\title{
Suppression of large edge localized modes with edge resonant magnetic fields in high confinement DIII-D plasmas
}

\author{
T.E. Evans, R.A. Moyer ${ }^{1}$, J.G. Watkins ${ }^{2}$, T.H. Osborne, P.R. Thomas ${ }^{3}$, \\ M. Becoulet ${ }^{3}$, J.A. Boedo ${ }^{1}$, E.J. Doyle ${ }^{4}$, M.E. Fenstermacher ${ }^{5}$, K.H. Finken ${ }^{6}$, \\ R.J. Groebner, M. Groth ${ }^{5}$, J.H. Harris ${ }^{7}$, G.L. Jackson, R.J. La Haye, C.J. Lasnier ${ }^{5}$, \\ S. Masuzaki $^{8}$, N. Ohyabu ${ }^{8}$, D.G. Pretty ${ }^{7}$, H. Reimerdes ${ }^{9}$, T.L. Rhodes ${ }^{4}$, \\ D.L. Rudakov ${ }^{1}$, M.J. Schaffer, M.R. Wade ${ }^{10}$, G. Wang ${ }^{4}$, W.P. West and L. Zeng ${ }^{4}$ \\ General Atomics, PO Box 85608, San Diego, CA 92186-5608, USA \\ ${ }^{1}$ University of California San Diego, La Jolla, California, USA \\ 2 Sandia National Laboratory, Albuquerque, New Mexico, USA \\ ${ }^{3}$ CEA-Cadarache Euratom Association, Cadarache, France \\ ${ }^{4}$ University of California, Los Angeles, California, USA \\ ${ }^{5}$ Lawrence Livermore National Laboratory, Livermore, California, USA \\ ${ }^{6}$ FZ-Jülich Euratom Association, Jülich, Germany \\ ${ }^{7}$ Australian National University, Canberra, Australia \\ ${ }^{8}$ National Institute for Fusion Science, Gifu-ken, Japan \\ ${ }^{9}$ Columbia University, New York, New York, USA \\ ${ }^{10}$ Oak Ridge National Laboratory, Oak Ridge, Tennessee, USA \\ E-mail: evans@fusion.gat.com
}

Received 9 December 2004, accepted for publication 5 May 2005

Published 24 June 2005

Online at stacks.iop.org/NF/45/595

\begin{abstract}
Large sub-millisecond heat pulses due to Type-I edge localized modes (ELMs) have been eliminated reproducibly in DIII-D for periods approaching nine energy confinement times $\left(\tau_{E}\right)$ with small dc currents driven in a simple magnetic perturbation coil. The current required to eliminate all but a few isolated Type-I ELM impulses during a coil pulse is less than $0.4 \%$ of plasma current. Based on magnetic field line modelling, the perturbation fields resonate with plasma flux surfaces across most of the pedestal region $\left(0.9 \leqslant \psi_{\mathrm{N}} \leqslant 1.0\right)$ when $q_{95}=3.7 \pm 0.2$, creating small remnant magnetic islands surrounded by weakly stochastic field lines. The stored energy, $\beta_{\mathrm{N}}, \mathrm{H}$-mode quality factor and global energy confinement time are unaltered by the magnetic perturbation. Although some isolated ELMs occur during the coil pulse, long periods free of large Type-I ELMs $\left(\Delta t>4-6 \tau_{E}\right)$ have been reproduced numerous times, on multiple experimental run days in high and intermediate triangularity plasmas, including cases matching the baseline ITER scenario 2 flux surface shape. In low triangularity, lower single null plasmas, with collisionalities near that expected in ITER, Type-I ELMs are replaced by small amplitude, high frequency Type-II-like ELMs and are often accompanied by one or more ELM-free periods approaching 1-2 $\tau_{E}$. Large Type-I ELM impulses represent a severe constraint on the survivability of the divertor target plates in future burning plasma devices. Results presented in this paper demonstrate that non-axisymmetric edge magnetic perturbations provide a very attractive development path for active ELM control in future tokamaks such as ITER.
\end{abstract}

PACS numbers: 28.52.-s, 52.55.Fa, 52.55.Rk

(Some figures in this article are in colour only in the electronic version)

\section{Introduction}

Edge localized modes (ELMs) are repetitive instabilities that often appear in the pedestal region of plasmas with edge transport barriers (H-mode plasmas). Although these instabilities play an important role in regulating the global plasma confinement and core impurity content, they also pose a significant threat to the integrity of plasma facing materials as reactor relevant conditions are approached [1]. Type-I ELMs produce a rapid loss of energy from the pedestal region that 
is known to result in large impulsive loads on divertor target plates. In future tokamaks the thermal energy released during these Type-I ELMs is expected to reach or exceed $20 \%$ of the pedestal energy and may be released on a sub-millisecond time scale thus significantly exceeding the $45 \mathrm{MJ} \mathrm{m}^{-2} \mathrm{~s}^{-1 / 2}$ [2] impulse ablation threshold of carbon divertor tiles.

Because of their potential for eroding divertor target plates, Type-I ELMs must be controlled in fusion reactors such as ITER [3]. The control method to be used must reduce the amplitude of energy impulses without significantly altering the pressure at the top of the pedestal, which is intimately linked to global confinement. It must also replace the impulsive thermal and particle transport driven by Type-I ELMs with a more benign mechanism (i.e. a lower amplitude, longer duration transport process) in order to avoid an uncontrolled increase in the core density or a detrimental accumulation of impurity ions in the core plasma. Several control techniques are being examined in the current generation of tokamaks including: (1) impurity seeding [4], (2) the injection of small pellets to trigger ELM-like events (i.e. ELM pace-making) [5] and (3) the use of resonant magnetic perturbations (RMPs) [6-9]. In this paper we focus on recent results using edge RMPs in DIII-D.

Based on linear ELM stability theory, relatively small changes in the outer pedestal pressure and current profiles can have a significant stabilizing effect on large Type-I ELMs. Thus, in principle, an edge stochastic layer, which is known to modify heat and particle transport across the pedestal [10] and to locally decrease the electron temperature gradient across the outer pedestal region [11], is of considerable interest as a potential tool for controlling ELMs and other naturally occurring transport processes across the pedestal of a fusion reactor. In addition to modifying the edge temperature profile, a stochastic layer formed across the peripheral pedestal region is expected to create a relatively cool buffer plasma, which should enhance impurity line radiation at the foot of the pedestal, as the collisionality and neutral ionization increase in this region. Active control of this buffer plasma could also be of value as a mechanism for radiatively spreading energy flux escaping from the core and pedestal plasma over a broad area of the divertor targets and wall. Recent stochastic boundary modelling and experimental data suggest that these peripheral stochastic layers are not incompatible with H-modes in poloidally diverted plasmas [12] and it was found earlier that edge transport barriers can form and be maintained with stochastic boundary layers under some conditions in limiter plasmas [13]. Therefore, it is not unreasonable to assume that an edge stochastic layer may well be capable of shifting the pedestal pressure profile inward and broadening it while altering the bootstrap current across the outer pedestal region, thus providing an effective ELM control mechanism. Here, it is noted that under some conditions, experimental data obtained in DIII-D provide strong support for the stochastic ELM suppression framework described earlier [8], but in other cases, such as for those discussed in this paper, the data suggest that another type of basic transport mechanism may prove equally valuable for ELM control in future devices.

\section{Experimental overview and results}

Dedicated Type-I ELM control experiments using edge RMPs were initiated in 2003 when DIII-D was first equipped with

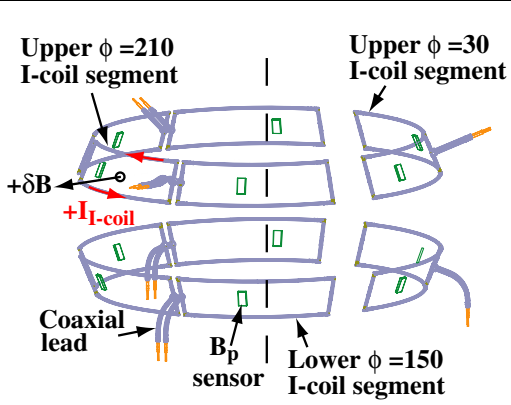

Figure 1. The I-coil comprises six segments above the equatorial plane (upper) and six segments below (lower).

internal magnetohydrodynamic (MHD) control coils designed for $n=1$ resistive wall mode (RWM) feedback control. Field line modelling of $n=3$ perturbations from these coils with the TRIP3D code [12] has shown that they are also reasonably well suited for producing modest edge stochastic layers with relatively small core perturbations.

A variety of ELM suppression and modification results, over a range of plasma shapes and conditions, have been obtained during these experiments in DIII-D. Nevertheless, this paper focuses on a limited set of results in which the perturbation coil was configured to produce multiple magnetic island chains on rational flux surfaces across the pedestal with a relatively narrow stochastic flux loss region $(\sim 3 \%$ in poloidal magnetic flux) just inside the unperturbed primary separatrix. The ELM suppression results found in this configuration are quite interesting from the perspective of physics because they leave the time-averaged electron pressure profile essentially unaltered, along with the global energy confinement time, the radial electric field profile and the poloidal rotation profile. At the same time, the $\mathrm{C}$ vi ion pressure profile shifts outwards when the toroidal rotation drops significantly. Thus, with this particular perturbation coil configuration, the experimental data does not fit within a conceptual framework in which a strongly enhanced stochastic field line transport at the foot of the pedestal flattens the pressure profile and leads to a stabilization of the large Type-I ELMs. Since the perturbation coil was not specifically designed to produce edge localized stochastic boundary layers (the poloidal mode spectrum is marginally suitable for this application) and has initially been limited to coil currents of $4.4 \mathrm{kA}$, it is not at all surprising that the stochastic flux loss region is relatively small in these experiments. Future experiments, at the full coil current design value of $7 \mathrm{kA}$, will significantly increase the width of the boundary layer stochastic flux loss region at the foot of the pedestal and are expected to provide valuable information on how changing this width affects the edge pressure profile and MHD stability as well as transport barrier physics.

\subsection{Description of the RMP coil}

The internal RWM control coil, referred to as the I-coil [14], is used as a controlled perturbation field source for the ELM suppression experiments. The three-dimensional geometry of the one-turn I-coil loops is shown in figure 1. Since the external C-coil, used to correct field-errors in the DIII-D core plasma, is also known to perturb the pedestal plasma [15] it was turned off for these experiments. 




Figure 2. The poloidal mode spectrum of the I-coil configured for $n=3$, odd parity, operation with $\pm 4.4 \mathrm{kA}$ in each coil segment.

In order to minimize core RMPs due to the I-coil, toroidally adjacent segments are driven with opposite polarities producing an $n=3$ toroidal mode structure. Positive current is defined to be in the counter-clockwise direction when viewed inward towards the toroidal axis and the corresponding perturbation field $(\delta B)$ points in the direction of positive major radius $R$ (see the upper segment at $210^{\circ}$ in figure 1 ). The up/down parity of the coil is referred to as 'odd', when the upper and lower coil segments have opposite polarities at each toroidal angle $\phi$ and the toroidal phase angle $\phi_{\mathrm{I} \text {-coil }}=0^{\circ}$ is defined by a positive current in the upper $30^{\circ}$ segment. The poloidal mode structure of the flux surface normal perturbation field component at $q=11 / 3$ has a pronounced notch at $m=11$ for the odd parity configuration as shown in figure 2 . On the $q=2$ surface the amplitude of the $6 / 3$ mode drops to $\sim 1.5 \mathrm{G}$ and the $3 / 3$, with $\sim 7.5 \mathrm{G}$, dominates the spectrum. For comparison, when the DIII-D C-coil is used to optimize error field correction, the resonant $m, n=2,1$ perturbation at the $q=2$ surface is larger, $\sim 7.5 \mathrm{G}$, than that from the $n=3$, odd parity I-coil. In the even parity configuration the peak in the poloidal mode spectrum moves up to $m=10$ and the notch moves down to $m=5$, producing a broader stochastic resonance structure across the pedestal.

\subsection{Description of discharge types}

Results from three types of lower single null (LSN) plasma configurations are discussed in this paper. The first is our reference ELM suppression shape having a modestly up-down asymmetric equilibrium biased downwards by $2.0 \mathrm{~cm}$. This shape, shown in figure 3 (115467), has an elongation $(\kappa)$ of 1.8 , upper $\left(\delta_{\text {up }}\right)$ and lower $\left(\delta_{\text {low }}\right)$ triangularities of 0.35 and 0.73 , respectively, a toroidal magnetic field $B_{\mathrm{T}}=1.6 \mathrm{~T}$, a plasma current $I_{\mathrm{p}}=1.1 \mathrm{MA}$, neutral beam heating power $\left(P_{\mathrm{inj}}\right)$ of 5.1 MW and an aspect ratio $(A)$ of 3.1. In these discharges, the line averaged electron density $\left(n_{\mathrm{e}}\right)$ was $6.9 \times 10^{19} \mathrm{~m}^{-3}$ with $\beta_{\mathrm{N}}=2.2, H_{\mathrm{L} 89}=2.1, \tau_{E}=160 \mathrm{~ms}$, a stored energy of $0.71 \mathrm{MJ}$ and a safety factor at the $95 \%$ flux surface $\left(q_{95}\right)$ of 3.8. The normalized pedestal density $n_{\mathrm{e}}^{\text {ped }} / n_{\mathrm{GW}}=0.57$ with a pedestal $\beta^{\text {ped }}=1.1 \%, \rho_{*}^{\text {ped }}=0.5 \%$ and an electron pedestal collisionality $\nu_{*}^{\text {ped }}=0.56$. The second configuration, shown in figure 3 (119690), is derived from an ITER equilibrium file provided by the ITER team [16] that is scaled down by a factor of 3.5 to fit into the DIII-D vacuum vessel. These plasmas have an elongation $(\kappa)$ of 1.8 , upper $\left(\delta_{\text {up }}\right)$ and lower $\left(\delta_{\text {low }}\right)$ triangularities of 0.43 and 0.60 , respectively, a toroidal magnetic field $B_{\mathrm{T}}=1.6 \mathrm{~T}$, a plasma current $I_{\mathrm{p}}=1.0 \mathrm{MA}$, neutral beam heating power $\left(P_{\mathrm{NBI}}\right)$ of $4.8 \mathrm{MW}$ and an $A$ of 3.1. In these discharges, the line averaged electron density $\left(n_{\mathrm{e}}\right)$

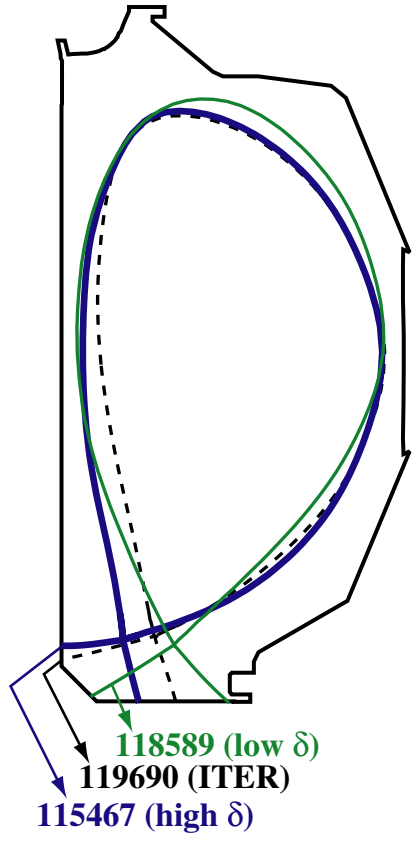

Figure 3. Comparison between discharge shapes used in the DIII-D ELM suppression experiments.



Figure 4. (a) $I_{\mathrm{p}}, P_{\mathrm{inj}}, I_{\mathrm{I}-\text { coil }}$ current and $(b) \mathrm{D}_{2}$ gas fuelling and line-averaged $n_{\mathrm{e}}$ in discharge 115467.

was $7.2 \times 10^{19} \mathrm{~m}^{-3}$ with $\beta_{\mathrm{N}}=2.0, H_{\mathrm{L} 89}=1.9, \tau_{E}=$ $160 \mathrm{~ms}$, a stored energy of $0.57 \mathrm{MJ}$ and a safety factor at the $95 \%$ flux surface $\left(q_{95}\right)$ of 3.7 . The normalized pedestal density $n_{\mathrm{e}}^{\text {ped }} / n_{\mathrm{GW}}=0.42$ with a pedestal $\beta^{\text {ped }}=0.43 \%$, $\rho_{*}^{\text {ped }}=0.45 \%$ and a pedestal collisionality $\nu_{*}^{\text {ped }}=0.79$. The final shape shown in figure 3 (118589) is a low triangularity configuration with the following parameters: $B_{\mathrm{T}}=1.9 \mathrm{~T}$, $I_{\mathrm{p}}=1.1-1.4 \mathrm{MA}, n_{\mathrm{e}}=4.0-4.8 \times 10^{19} \mathrm{~m}^{-3}, \kappa=1.8$, $\delta_{\text {low }}=0.38, P_{\mathrm{NBI}}=13.1 \mathrm{MW}, \beta_{\mathrm{N}}=2.4, H_{\mathrm{L} 89}=1.8,3.3 \leqslant$ $q_{95} \leqslant 3.9$. The normalized pedestal density $n_{\mathrm{e}}^{\text {ped }} / n_{\mathrm{GW}}=0.14$ with a pedestal $\beta^{\text {ped }}=0.46 \%, \rho_{*}^{\text {ped }}=0.62 \%$ and a pedestal collisionality $\nu_{*}^{\text {ped }}=0.03$. Note that this discharge has a plasma current ramp during the I-coil pulse that produces a $q_{95}$ scan across the peak in the resonant window of the perturbation coil and beta feedback using NBI heating is turned on to maintain a constant normalized beta $\beta_{\mathrm{N}}$ level.

The time evolution of $I_{\mathrm{p}}, P_{\mathrm{inj}}, n_{\mathrm{e}}$, the $\mathrm{D}_{2}$ gas fuelling and the I-coil pulse $\left(I_{\text {coil }}=4.4 \mathrm{kA}\right)$ is shown for our reference ELM suppression discharge (115467) in figure 4, for an ITER scenario 2 discharge (119690) in figure 5 and for a low triangularity discharge (118589) in figure 6. Reproducible ELM suppression has also been obtained in the reference shape with $B_{\mathrm{T}}=2.0 \mathrm{~T}$ and $I_{\mathrm{p}}=1.4 \mathrm{MA}\left(q_{95}=3.8\right)$ as well as with $B_{\mathrm{T}}=2.0 \mathrm{~T}$ and $I_{\mathrm{p}}=1.1 \mathrm{MA}\left(q_{95}=4.9\right)$. 




Figure 5. (a) $I_{\mathrm{p}}, P_{\mathrm{inj}}$ (modulation shown as thick black regions) and $I_{\mathrm{I} \text {-coil }}$ current and $(b) \mathrm{D}_{2}$ gas fuelling and line-averaged $n_{\mathrm{e}}$ in discharge 119690 (the ITER scenario 2 shape).

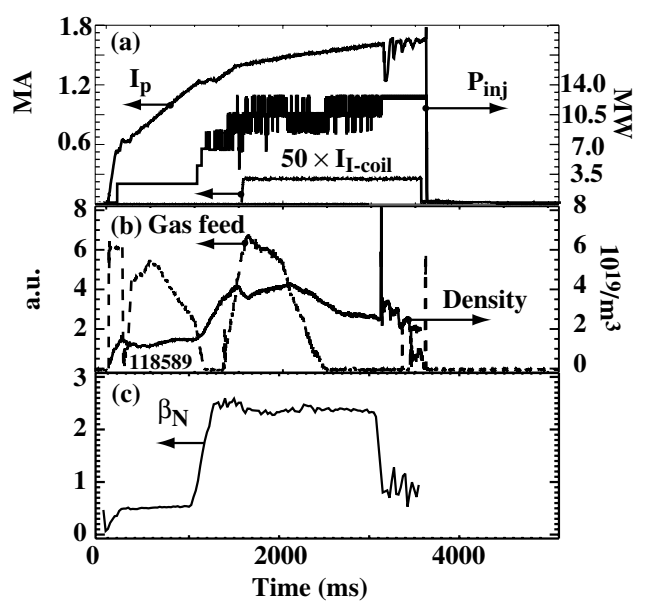

Figure 6. (a) $I_{\mathrm{p}}, P_{\text {inj }}$ (modulation shown as thick black regions) and $I_{\mathrm{I} \text {-coil }}$ current, $(b) \mathrm{D}_{2}$ gas fuelling and line-averaged $n_{\mathrm{e}}$ and (c) normalized beta in discharge 118589 .

\subsection{Experimental results from high triangularity $L S N$ reference shapes}

A notable characteristic of good ELM suppression is a global change in the dynamics of the $\mathrm{D}_{\alpha}$ recycling light measured at various toroidal and poloidal locations. Well-correlated changes in all the divertor diagnostics typically used to monitor the properties of the ELMs are also seen. An example of the change seen in the divertor $\mathrm{D}_{\alpha}$ ELM dynamics when the I-coil is applied to a discharge in the reference shape is shown in figure 7. Here, a discharge with an $n=3$ I-coil pulse (115467) is compared with a discharge without an I-coil pulse (115468). Similar changes are also observed in the midplane $\mathrm{D}_{\alpha}$ signals at $\phi=45^{\circ}$, all of the lower divertor $\mathrm{D}_{\alpha}$ signals at $\phi=135^{\circ}$, all of the upper divertor $\mathrm{D}_{\alpha}$ signals at $\phi=150^{\circ}$, the inner wall $\mathrm{D}_{\alpha}$ at $\phi=135^{\circ}$ and the lower divertor surface temperature measured by an infrared TV (IRTV) at $\phi=165^{\circ}$. Changes consistent with Type-I ELM suppression are also seen in the core $B_{\mathrm{dot}}$ signals at the outer midplane, $\phi=322^{\circ}$, and in the edge $B_{\text {dot }}$ signal from the divertor at $\phi=322^{\circ}$. Fixed Langmuir probes in the lower divertor at $\phi=180^{\circ}$, measuring ion saturation current, also see a reduction in the particle flux consistent with the suppression of Type-I ELMs during the I-coil pulse.

Each of these diagnostics shows three well-defined characteristics during the ELM suppression phase. Large

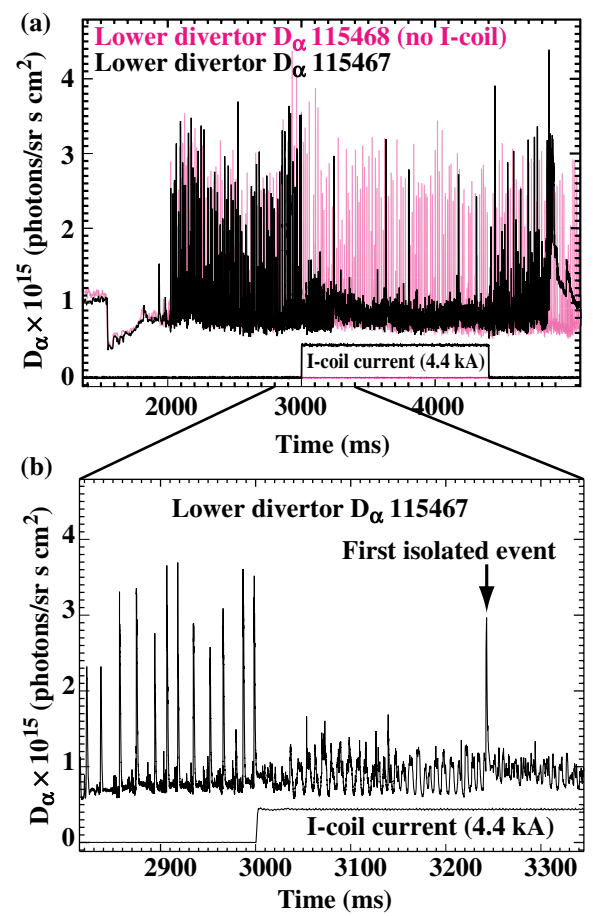

Figure 7. (a) Lower divertor $\mathrm{D}_{\alpha}$ signal near the outer strike point showing Type-I ELM suppression in discharge 115467 (black) during a $4.4 \mathrm{kA} I_{\mathrm{I} \text {-coil }}$ pulse compared with an identical discharge, 115468 (magenta), without an I-coil pulse. (b) A shorter time window showing a change in the dynamics across the I-coil turn on time for the Type-I ELM suppression discharge only.

spikes owing to Type-I ELMs are suppressed within a single $15 \mathrm{~ms}$ ELM period, small $130 \mathrm{~Hz}$ coherent oscillations with a $2 \mathrm{~ms}$ quiet period and a $6 \mathrm{~ms}$ active period are observed between intermittent Type-I ELM-like events, and when the perturbation field is turned off large Type-I ELMs return. When the I-coil current is first switched on at $t=3000 \mathrm{~ms}$ there is a short $\Delta t \sim 20-30 \mathrm{~ms}$ period of small incoherent fluctuations. Although this activity looks remarkably similar to the behaviour seen in the discharge without the I-coil pulse (115468), large Type-I ELMs are immediately suppressed. A first indication of the onset of coherent oscillations is observed at $\sim 3040 \mathrm{~ms}$, and intermittent Type-I ELM-like events, such as the one shown at $t=3243 \mathrm{~ms}$, sometimes punctuate the oscillations. Note that the oscillations become rather chaotic after the isolated event but eventually recover their coherent structure. Subsequent isolated events have similar effects on the oscillations. Near the foot of the pedestal, the toroidal rotation, which has been decreasing since the I-coil current was switched on, reaches zero at roughly the same time as these oscillations first appear. Deeper inside the plasma and well inside the pedestal region, the toroidal rotation drops on a slower time scale reaching approximately one-third of its initial value by $t=3300 \mathrm{~ms}$.

It is remarkable, as shown in figure 8 , that the core confinement is unaltered with such dramatic changes in the edge MHD and toroidal rotation and interesting to note that the electron pedestal pressure increases modestly, whereas, except for a random few isolated spikes, large Type-I ELMs are almost entirely eliminated. The radial electron pedestal profiles measured with Thomson scattering and ion pedestal 


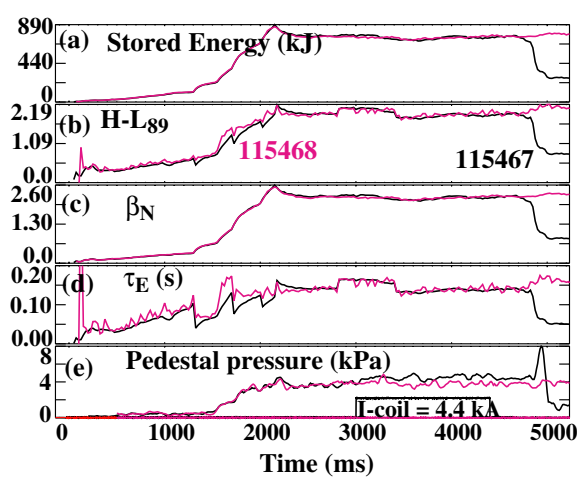

Figure 8. (a) Stored energy, (b) H-factor $\left(\mathrm{H}-\mathrm{L}_{89}\right),(c)$ beta normal, $(d)$ energy confinement time and $(e)$ electron pedestal pressure in discharge 115467 (black) during a $4.4 \mathrm{kA} I_{\text {I-coil }}$ pulse compared with an identical discharge, $115468(t=0$ to $>5000 \mathrm{~ms})$, without an I-coil pulse.

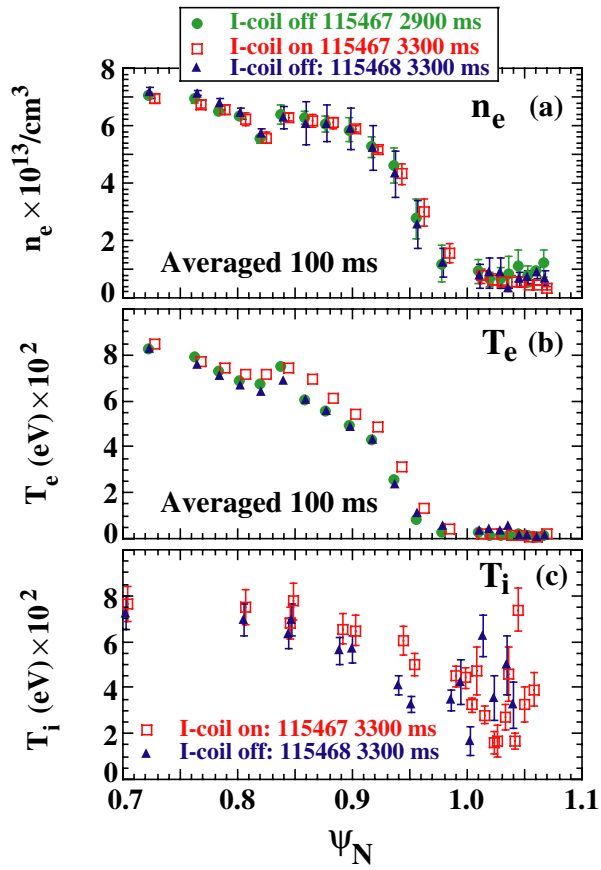

Figure 9. (a) $n_{\mathrm{e}}$ and (b) $T_{\mathrm{e}}$ averaged over $100 \mathrm{~ms}$ before the I-coil pulse (solid dots) and during the I-coil pulse (open square) in discharge 115467 compared with the reference discharge (115468) without an I-coil pulse (solid triangles) versus normalized poloidal magnetic flux $\psi_{\mathrm{N}}$. In (c) $T_{\mathrm{i}}$ is shown in discharge 115467 during the I-coil pulse (red) and compared with $T_{\mathrm{i}}$ during the reference discharge (115468) with no I-coil (blue).

$T_{\mathrm{i}}$ profiles measured with charge exchange spectroscopy are averaged over $100 \mathrm{~ms}$ before and during the I-coil pulse as well as during the reference discharge without the I-coil pulse (115468) and are displayed in figure 9. These profiles remain relatively constant during the suppressed ELM phase when compared with those during the ELMing phase.

A key feature of the coherent oscillations that replace the ELMs is that they appear to result from a process that produces about the same time-averaged transport through the pedestal as is seen during the Type-I ELMing phase, but without the large impulsive component typical of the Type-I ELMs. This is attributed to the longer active period and smaller amplitudes of these structures compared with the rapid Type-I

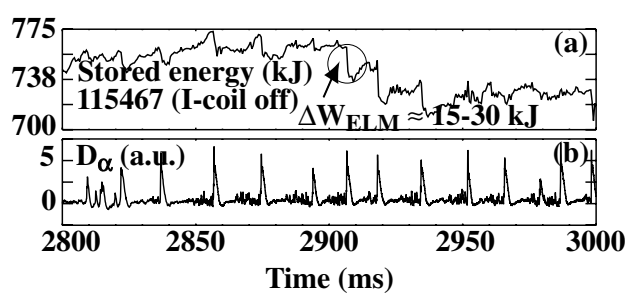

Figure 10. (a) Changes in stored energy associated with each ELM, (no I-coil current). (b) A lower divertor $\mathrm{D}_{\alpha}$ signal (100 $\mu$ s resolution).



Figure 11. (a) The fast stored energy signal as in figure $10(a)$ but during the I-coil pulse and $(b)$ oscillations in the lower divertor $\mathrm{D}_{\alpha}$ signal.

ELM spikes. Similar effects are observed in the stored energy calculated from EFIT equilibrium reconstructions with time steps of $\Delta t_{\mathrm{WMHD}}=500 \mu \mathrm{s}$. During the ELMing phase, Type-I ELMs transport as much as $30 \mathrm{~kJ}$ of the pedestal energy into the Scrape-Off Layer (SOL) during one $\Delta t_{\mathrm{WMHD}}$ as shown by the largest drops in figure 10(a) (figure 10(b) shows lower divertor $\mathrm{D}_{\alpha}$ spikes associated with drops in the stored energy). Midplane diagnostics indicate that this energy is expelled within $200 \mu$ s or less. Since the magnitude of the drop can be somewhat underestimated by the $\Delta t_{\mathrm{WMHD}}$ step in the EFIT calculation (because of the inherent time resolution of the diagnostics used to calculate the stored energy using the EFIT code), this implies a minimum impulsive source $\left(W_{\text {imp }} \equiv \Delta W_{\mathrm{ELM}} / \Delta t^{1 / 2}\right)$ into the SOL for the largest ELMs of $W_{\mathrm{imp}}=2.10 \mathrm{MJ} \mathrm{s}^{-1 / 2}$. During the I-coil pulse, the stored energy drops are considerably smaller and slower as shown in figure 11 $(a)$ (with the associated lower divertor $\mathrm{D}_{\alpha}$ oscillations shown in figure $11(b)$ ). The largest of these are of order $15 \mathrm{~kJ}$ and evolve over $\sim 500 \mu \mathrm{s}$, implying a maximum impulsive source of $W_{\text {imp }}=0.67 \mathrm{MJ} \mathrm{s}^{-1 / 2}$ or a factor of 3 reduction. Although such estimates are informative, they do not address such key issues as to how much of the energy lost from the pedestal reaches the divertor targets and main chamber walls or how the energy is distributed over these plasma facing surfaces. Our data show that the energy lost from the pedestal is dispersed over a larger area by the perturbed magnetic topology.

In DIII-D, fixed Langmuir probes and infrared (IR) cameras measure heat and particle flux to the divertor target plates owing to ELM impulses. These diagnostics are used to determine the absolute magnitudes of heat and particle flux impulses striking plasma facing surfaces. An example of this is given in figure 12, where fast radial line scan data from an IR camera viewing the lower DIII-D divertor is shown averaged over five ELM peaks (figure 12(a)) before the I-coil pulse and four peaks during the active phase of the $130 \mathrm{~Hz}$ 


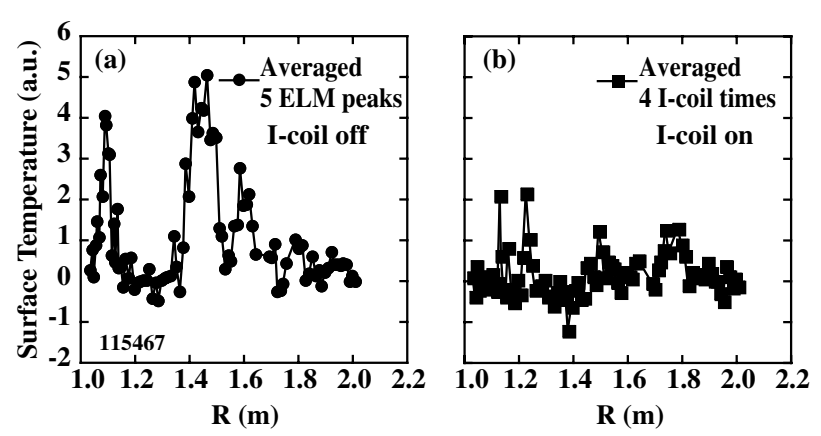

Figure 12. (a) IRTV line scans $(100 \mu \mathrm{s})$ of the lower divertor surface temperature with the I-coil off and $(b)$ with the I-coil on.



Figure 13. A lower divertor Langmuir probe signal near the outer strike point without the I-coil (upper) and with the I-coil (lower).

oscillations (figure 12(b)). Here, the stationary background signal (which is related to the target plate surface temperature) is subtracted from the fast changes and given in arbitrary units (a.u.), since calibrated measurements are not available in this mode of operation. A full radial scan of the lower divertor target plates is acquired every $100 \mu$ s and those scans with peaks corresponding to Type-I ELM or the active phase of the $130 \mathrm{~Hz}$ oscillations are selected and averaged to produce this plot. In figure 12(a), I-coil off, two significant peaks near the inner $(R=1.1 \mathrm{~m})$ and outer $(R=1.4 \mathrm{~m})$ strike points are clearly defined. A third peak is present at $R=1.6 \mathrm{~m}$ but this feature does not substantially exceed the noise level of the camera (1-2 a.u.). With the I-coil on, averages over the largest active phase oscillation peaks, figure 12(b), do not reveal any significant features. These data indicate that the heat flux driven by Type-I ELM is reduced by at least a factor of 5 during the I-coil pulse.

In general, the divertor Langmuir probes see a reduction in the impulsive particle flux $\left(\Gamma_{\text {imp }}\right)$ driven by Type-I ELMs, although probes at some locations see smaller reductions than others. Langmuir probe data acquired from discharges in the reference shape (115464 and 115467) show that the largest Type-I ELM driven particle flux impulses often reach $\Gamma_{\text {imp }}=$ $\Delta \Gamma / t^{1 / 2}=4 \mathrm{kA} \mathrm{cm}^{-2} \mathrm{~s}^{-1 / 2}$ near the outer strike point in the lower divertor. During the I-coil pulse, excluding isolated ELM-like events discussed earlier, these particle flux impulses are reduced to $\sim 0.8 \mathrm{kA} \mathrm{cm}^{-2} \mathrm{~s}^{-1 / 2}$ or a factor of 8 reduction. An example of the particle flux evolution is given in figure 13, where an oscillation peak during the I-coil pulse (black) is plotted relative to a Type-I ELM structure seen near the outer strike point before the I-coil is pulsed. For comparison,



Figure 14. (a) Lower divertor $\mathrm{D}_{\alpha}$ signal showing long ELM suppression phase during the I-coil pulse in discharge 119690 and (b) change in the baseline recycling response showing an increase in incoherent recycling fluctuations between several small ELMs that persist during the first $60-70 \mathrm{~ms}$ of the I-coil pulse.

referring to the $\mathrm{D}_{\alpha}$ signal in figure 6 during discharge 115467 (digitized at $100 \mathrm{kHz}$ ), the implied recycling impulse due to the ELM at $t=2998 \mathrm{~ms}$ is $\mathrm{D}_{\alpha \_ \text {imp }}=3.0 \times\left(10^{16}\right.$ photons $/ \mathrm{sr} \times$ $\left.\mathrm{cm}^{2} \mathrm{~s}\right) /(60 \mu \mathrm{s})^{1 / 2}=3.9 \times 10^{18}$ photons $/ \mathrm{sr} \times \mathrm{cm}^{2} \mathrm{~s}^{3 / 2}$. This is a factor of 3.1 larger than that owing to the largest feature $\mathrm{D}_{\alpha \_ \text {imp }}=0.8 \times\left(10^{16}\right.$ photons $\left./ \mathrm{sr} \times \mathrm{cm}^{2} \mathrm{~s}\right) /(40 \mu \mathrm{s})^{1 / 2}=1.3 \times$ $10^{18}$ photons $/ \mathrm{sr} \times \mathrm{cm}^{2} \mathrm{~s}^{3 / 2}$ seen with the I-coil on.

Signals from a midplane reciprocating Langmuir probe located $\sim 4 \mathrm{~cm}$ outside the unperturbed separatrix also show a suppression of the Type-I ELM impulses and an increase in higher frequency fluctuations that fill in the periods between the ELM crashes before the I-coil pulse. The nature of these higher frequency components is still under investigation but they appear to be very similar to the behaviour seen in the midplane reflectometer and edge magnetic probe data and suggest that the I-coil perturbation is effectively opening up a higher frequency transport channel, which moderates the buildup of the pedestal conditions needed for triggering large Type-I ELMs.

In discharges with exactly the same shape and input power as our high triangularity reference shape (115467 discussed earlier), but with slightly higher pedestal collisionality and $\beta_{\mathrm{N}}$, there is generally a modest increase in the $n=$ odd core MHD level and the response to the I-coil pulse has a tendency to behave more like the active periods of the $130 \mathrm{~Hz}$ oscillations, discussed earlier, without the associated quiet periods seen in those somewhat lower pedestal collisionality cases. The response to the I-coil pulse in one of these higher electron collisionality and $\beta_{\mathrm{N}}$ discharges (i.e. discharge 119390 with $\nu_{*}^{\text {ped }}=0.79$ and $\beta_{\mathrm{N}}=2.4$ ) is shown in figure 14 . Note that the large Type-I ELMs are not suppressed quite as rapidly in this case but are eventually stabilized for a relatively long period $\left(\Delta t=8.6 \tau_{E}\right.$ or $\sim 1300 \mathrm{~ms}$ ). In figure $14(b)$ we see that the ELMs are initially smaller when the I-coil is switched on and the baseline activity between these smaller ELMs increases. After about $60-70 \mathrm{~ms}$ the small ELMs are completely stabilized and then there are two large Type-IELMs followed by the long 8.6 $\tau_{E}$ ELM-free period.

Safety factor scans were carried out in these high triangularity reference shapes to determine the extent of the resonant ELM suppression window. Shown in figure 15 is 




Figure 15. (a) Lower divertor $\mathrm{D}_{\alpha}$ signal showing ELM suppression and re-appearance of ELMs during the I-coil pulse with a $q_{95}$ scan in discharge 115472 , (b) change in $q_{95}$ as $I_{\mathrm{p}}$ is increased during the discharge and $(c)$ the timing of the I-coil current pulse relative to the $q_{95}$ scan.

the result of one such scan. Here the plasma current was slowly increased starting at $t=3.3 \mathrm{~s}$ and $q_{95}$ decreased linearly in time from 3.8 to 3.3 during the I-coil current pulse as shown in figures $15(b)$ and $(c)$. We see in the lower divertor $\mathrm{D}_{\alpha}$ signal shown in figure $15(a)$ that the ELMs are initially suppressed when the I-coil current is turned on at $3.0 \mathrm{~s}$ and that there are a few isolated events over the next $0.7 \mathrm{~s}$ as $q_{95}$ begins to drop. As $q_{95}$ approaches 3.6-3.5, we see an increase in the baseline activity indicating the return of small ELMlike features. By the time $q_{95}$ reaches 3.5 these features are a predominant part of the divertor recycling dynamics and their amplitude grows as $q_{95}$ continues to drop. By the end of the I-coil current pulse large Type-I ELMs have returned and the perturbation field appears to be only increasing the small ELM-like activity between the large Type-I ELMs as can be seen when these features disappear as the I-coil current is switched off. A series of discharges were run with similar $q_{95}$ scans starting from higher initial values of $q_{95}$. Using these data we have established that the resonant window for ELM suppression in the reference shape and with the pedestal parameters given above for this reference shape extends from $q_{95} \sim 3.9$ down to $\sim 3.5$. While other isolated resonance windows at higher $q_{95}$ have also been observed, the one at 3.7 has consistently produced good suppression results. Of course, the range and position of this resonant window is expected to change with the shape of the discharge [12] and the pedestal parameters used as discussed later.

Variations made during experiments in the reference shape also demonstrate that changes in the triangularity, squareness and up-down symmetry have a significant impact on both the duration of the ELM suppression phase and the magnitude of the impulse reductions obtainable.

\subsection{Experimental results in an ITER scenario 2 shape}

The ITER scenario 2 shape (as shown in figure 3 ) is of particular interest with respect to this question since it is the one planned for $Q=10$ ELMing H-mode operations. Although the pedestal profile parameters expected in ITER (e.g. $\beta, \rho_{*}, v_{*}$ ) cannot be obtained in DIII-D, the flux surface shape has been closely reproduced in these experiments and good Type-I ELM suppression, qualitatively equivalent to those found in the

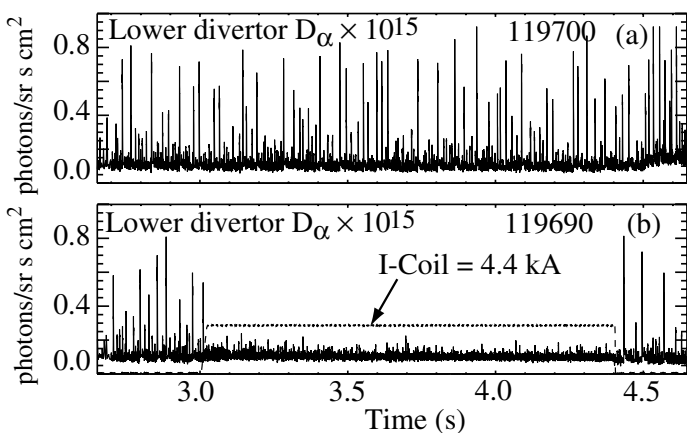

Figure 16. (a) Lower divertor $\mathrm{D}_{\alpha}$ signal near the outer strike point showing Type-I ELMs in an ITER scenario 2 discharge 119700 without an I-coil current pulse and $(b)$ a discharge with the same shape and pedestal parameters as that shown in (a) but with a $4.4 \mathrm{kA}$ I-coil pulse between 3.0 and $4.4 \mathrm{~s}$ (discharge 119690).

reference shape discussed earlier, has been achieved. An example of this is shown in figure 16, which may be compared with results from the reference shape shown in figure 7.

In the discharge without the I-coil current pulse (119700), the Type-I ELMs are not as regular as those in our reference discharge shape (115467). This change in the behaviour of the Type-I ELMs may be because of the differences in separatrix shape (e.g. lower triangularity) compared with the reference discharge shape (115467) as shown in figure 3. In the ITER scenario 2 shape we typically observe small sporadic ELMlike bursts between the large Type-I ELMs. These small features have some similarities with Type-II ELMs previously observed in DIII-D. When the I-coil current is switched on at $t=3.0 \mathrm{~s}$ the large Type-I ELMs are immediately suppressed but the smaller sporadic bursts are essentially unchanged. As with the active phase of the $130 \mathrm{~Hz}$ oscillation seen in the reference shape (115467), the small sporadic bursts do not have a significant impact on the stored energy and do not, in general, cause significant heat or particle flux impulses on the divertor target plates. We also note that in these discharges the core MHD activity, particularly those with $n=$ even MHD modes, was higher than that in our reference shape cases (e.g. discharges 115467 and 119390) and our best ELM suppression case was obtained with $\nu_{*}^{\text {ped }}=0.79$ and a small continuous deuterium gas feed as shown in figure 5. Although it was difficult to expand the ELM suppression window in discharges with this shape, we suspect that this result is an artefact of the limited I-coil current available during these experiments.

\subsection{Experimental results from a low triangularity LSN shape}

Since the reference and ITER scenario 2 shapes discussed earlier are not well suited to pumping with the lower divertor cryopump, it is of interest to explore the response of the plasma to I-coil pulses when the strike point is pulled out towards the pump baffle as shown for discharge 118589 in figure 3. This change in shape results in a significantly lower triangularity, $\delta_{\text {low }}=0.38$, compared with our reference shape with $\delta_{\text {low }}=$ 0.73 and a substantial reduction in the pedestal collisionality owing to the lower densities obtained with the pumping and higher neutral beam heating powers run in this shape. With these operating conditions we have obtained electron pedestal 
collisionalities equivalent to those anticipated in ITER (e.g. $\nu_{*}^{\text {ped }}=0.03$ in discharges 118589 and 118590 discussed here). Based on theoretical arguments for the relative effectiveness of a stochastic boundary layer in tokamaks, we expect that the impact of the I-coil at the foot of the pedestal will increase in plasmas with lower collisionality since parallel transport will dominate cross-field transport in this case.

As shown in figure 6, the evolution of the plasma parameters in these discharges is relatively complex. The plasma current and $q_{95}$ are changing continuously during the I-coil pulse, the NBI power is being regulated via a feedback loop to maintain a constant $\beta_{\mathrm{N}}$, there is a relatively large deuterium gas puff during the first half of the I-coil pulse and the C-coil is being used in error-field feedback mode. In these discharges, $\beta_{\mathrm{N}}$ feedback is used to obtain high-power plasmas while avoiding RWMs. Although differences in the I-coil resonance window can be affected by flux surface shifts with increasing $\beta_{\mathrm{N}}$, we expect this to be a second order effect when compared with differences in pedestal collisionality and plasma shape. As discussed in [9], the response of the ELMs to the I-coil current pulse is also more complex than in the reference shape discussed earlier. During the initial part of the I-coil pulse it appears that the Type-I ELMs are reduced in amplitude by about an order of magnitude and the frequency doubles. Since there is a strong gas puff during the first half of the I-coil current pulse and $q_{95}$ is ramping down from 3.9 to 3.5 over the same period, it is more difficult to characterize the ELM response during this time in these discharges. In particular, the ELMs appear to be affected differently at various poloidal locations although we generally see a reduction in the ELM amplitude and an increase in the frequency of the remaining ELMs. Near the outer strike point in the lower divertor, at the entrance to the cryopump duct, the large gas puff tends to saturate the $\mathrm{D}_{\alpha}$ signal both with and without the I-coil and therefore it has not been possible to determine how the amplitude of the ELMs is affected at this location, although we do see an increase in the frequency which is consistent with the frequency seen at other locations. We refer to this behaviour as ELM modification rather than suppression [9] since it appears to affect the ELM dynamics locally rather than globally.

More generally, the Type-I ELMs observed in these lowcollisionality, high-power discharges have large impulsive $\mathrm{D}_{\alpha}$ recycling characteristics, typical of those seen in DIII-D and other poloidally diverted tokamaks, as the pedestal collisionality is reduced. The ELMs are substantially more conductive in nature in these discharges, affecting almost equally the $T_{\mathrm{e}}$ and $n_{\mathrm{e}}$ pedestal profiles, than the Type-I ELMs discussed earlier in the reference and ITER shapes with higher pedestal collisionalities. In the high collisionality cases, the Type-I ELM perturbation primarily affects the $n_{\mathrm{e}}$ pedestal with little or no effect on the $T_{\mathrm{e}}$ pedestal and, thus, are more convective in nature. An example of the Type-I ELM behaviour in the lower divertor of these low collisionality discharges is shown in figure $17(a)$, where both the I-coil and C-coil were switched off to establish a reference ELM behaviour for comparisons with the ELM modification/suppression discharges carried out in this shape. In figure $17(b)$ we show how the divertor recycling dynamics change during a combined $n=3$ I-coil and $n=1$ C-coil pulse
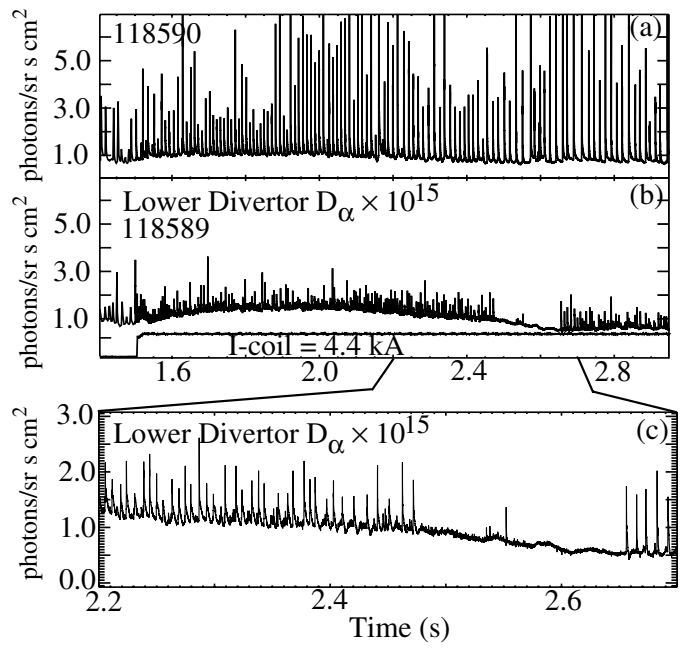

Figure 17. (a) Lower divertor $\mathrm{D}_{\alpha}$ signal showing Type-I ELM in a low triangularity reference discharge (118590) without an I-coil pulse compared with $(b)$ a similar discharge with an I-coil pulse and (c) an expanded view of ELM modification $(2.2 \leqslant t \leqslant 2.47)$ and ELM suppression phase in the discharge with the I-coil pulse (118589).

(the C-coil is configured to operate in the error field mode such that the current in the $\mathrm{C}$-coil tracks that of the poloidal field coils as the plasma current increases). In figure 17(b) we see that the Type-I ELMs are significantly reduced in amplitude during the perturbation coil pulse (I- and C-coil combined starting at $\sim 1500 \mathrm{~ms}$ shortly after the $\mathrm{L}-\mathrm{H}$ transition). As shown in [9], these small features have all the dynamics of the original Type-I ELMs but are about an order of magnitude smaller with twice the frequency.

We also note a particularly interesting period during the magnetic perturbation pulse starting at $t \sim 2.5 \mathrm{~s}$, where all the $\mathrm{D}_{\alpha}$ recycling signals around the machine shift to a relatively quiet state. During this period we see a global change in the pedestal as well as the divertor dynamics and Type-I ELMs are strongly suppressed (except for a set of isolated events between 2.535 and $2.552 \mathrm{~s}$ ). Although the pedestal properties observed during this period have some features that are similar to those seen during ELM suppression phases in our reference discharge shape discussed earlier, there are also significant differences. The most fundamental of these is that the ELM suppression period in these discharges spans a $q_{95}$ interval just below the resonant window required for good ELM suppression in the reference shape, i.e. $3.4 \leqslant$ $q_{95} \leqslant 3.5$. This difference in the resonant $q_{95}$ window is expected to be partly due to a shift in the effective mode structure of the I-coil as a result of a mixing with the Ccoil spectrum and to the lower triangularity shape of this discharge [12]. One interesting aspect of these relatively quiet ELM-free periods is that they turn-on rather abruptly just as the gas puff turns off and they have features that are quite similar to those found in QH-modes (although these discharges have co-injected NBI heating, whereas QH-modes have been observed only in DIII-D with counter-injection NBI heating). These quiet phases have pedestal profiles that are similar to those in QH-modes and do not show a buildup of the core or pedestal parameters typical of other ELM-free H-modes. Although they do not appear to have strong edge harmonic 


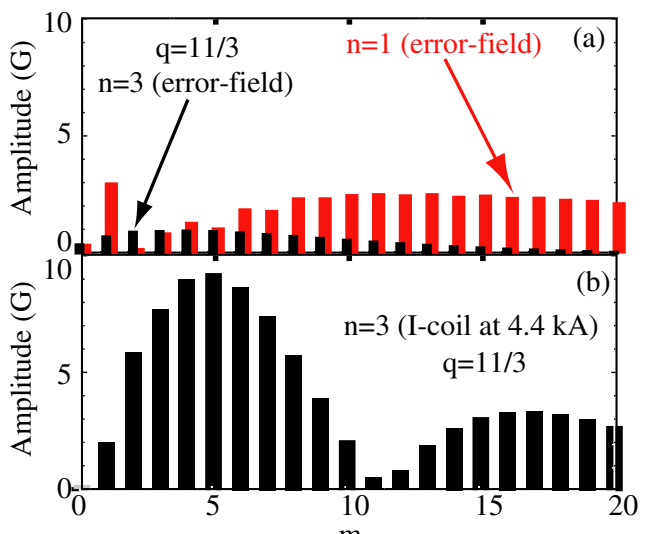

Figure 18. (a) Poloidal mode spectrum of the measured $n=1$ and $n=3$ intrinsic field-errors in DIII-D at the $q=11 / 3$ surface for the reference discharge shape discussed in section 2.3 compared with (b) the I-coil perturbation spectrum on the same $q$ surface with a current of $4.4 \mathrm{kA}$ (from figure 2).

mode signatures, which is typical of many QH-modes in DIII$\mathrm{D}$, they do occur in about the same parameter regimes as $\mathrm{QH}-$ modes and have particularly high ion temperatures with clear signatures of internal transport barriers that are seen in DIII-D quiescent double barrier (QDB) modes. These observations are particularly appealing since they suggest that there may be a connection between the ELM suppression physics operating in $\mathrm{QH}$-modes and those responsible for the suppression of ELMs in the rather disparate shape and collisionality regimes discussed in this paper. Additionally, it is noted that previous modelling studies of changes in the edge magnetic topology produced by the $\mathrm{C}$-coil showed that flat regions near the foot of some QH-mode profiles are in very good agreement with the width of the stochastic flux loss region calculated in the TRIP3D field line integration code [12].

\section{Field line modelling}

Field line modelling is an important tool in understanding how the pedestal magnetic topology changes with various magnetic perturbation sources and plasmas equilibrium shapes. In this section we describe how the magnetic structure of the pedestal changes, when compared with only the DIII-D field-errors, during the application of $n=3$, odd parity, I-coil pulses in the high triangularity reference shape discharges discussed in section 2.3. Although the axisymmetric equilibrium field used in this modelling contains information owing to the plasma pressure and current, it should be noted that the field line integration code does not account for the plasma response to the change imposed by the external magnetic perturbations, e.g. changes in the edge current or edge and core MHD modes. Nevertheless, it does provide essential information for a better understanding of the mechanisms responsible for controlling the pedestal dynamics discussed in the previous section of this paper and provides a starting point for future modelling work designed to account for the plasma response observed during these experiments.

It is important to realize that the magnitude of the perturbation applied during the $n=3$, odd parity, I-coil current pulses in our reference discharge shapes discussed

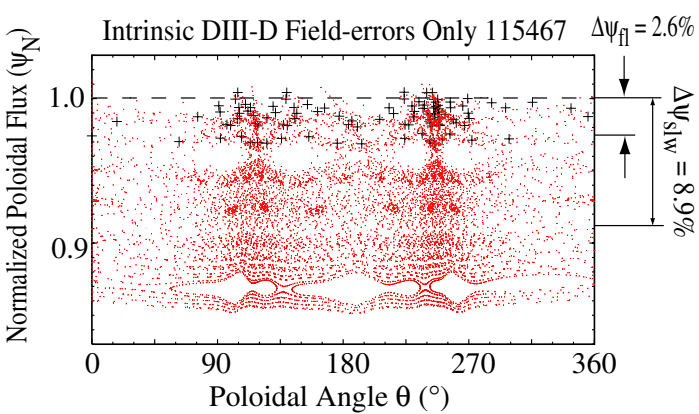

Figure 19. Rectangular Poincaré plot displaying the effect of measured DIII-D field-errors without the I-coil current perturbation during discharge 115467 (the reference high triangularity shape discussed earlier). The positions of the field lines (small dots) are plotted as a function of normalized poloidal magnetic flux $\psi_{\mathrm{N}}$ and poloidal angle $\theta$ across the pedestal region at $\phi$ (toroidal angle) $=0^{\circ}$. The black +'s show location of field lines that are lost to the wall in less than 200 toroidal transits.

earlier are about the same size as the measured field-errors in DIII-D. The relative amplitudes of the measured $n=1$ and $n=3$ field-errors at the $q=11 / 3$ surface are shown in figure 18(a) and the mode spectrum produced by the I-coil (from figure 2 earlier) is shown for comparison in figure $18(b)$ on the $q=11 / 3$ surface. Since this spectrum is a function of the distance from the perturbations source and we need to know its structure across the entire pedestal to interpret the experimental results, we find that it is much more informative to calculate the three-dimensional topology of the field lines using a field line integration code [12]. The field line trajectories calculated with this code are displayed in a rectangular, Poincaré surface-of-sections format, where the radial variable (the ordinate) is the normalized poloidal magnetic flux $\psi_{\mathrm{N}}$ and the angular variable (the abscissa) is the poloidal angle $\theta$. Each point on this plot represents the $\left(\psi_{\mathrm{N}}, \theta\right)$ position of a field line as it passes through the specified poloidal plane. These plots contain a tremendous amount of information about the magnetic structure of the pedestal region as can be seen in figure 19, where the effects of field-errors only (I-coil perturbations are not included in this calculation), in our reference discharge shape (115467) discussed earlier, are shown across the pedestal region of the plasma. Of particular interest in these plots are the width of the stochastic magnetic flux loss region, noted as $\Delta \psi_{\mathrm{fl}}$ in figure 19 , and the width of the stochastic field line mixing region noted as $\Delta \psi_{\text {slw }}$ in figure 19. The inner boundary of the stochastic flux loss region is defined by the deepest penetration, in $\psi_{\mathrm{N}}$, from which field lines are lost across the separatrix, whereas the stochastic mixing region contains field lines that move radially across unperturbed flux surfaces but do not escape across the separatrix. In the flux loss region some, but not necessarily all, of the field lines cross the location of the unperturbed separatrix and strike the divertor target plates. An example of such a field line is shown in figure 19 by the large ' + ' symbols. In this case, the field line makes 94 toroidal transits before being lost to the divertor target and $\Delta \psi_{\mathrm{fl}}=2.6 \%$. We note that not all the field lines in this region are connected to material surfaces (after 200 toroidal transits used in these calculations) and that as the perturbation level increases, the fraction of 'open' field lines (i.e. those connected to material surfaces) generally increases 


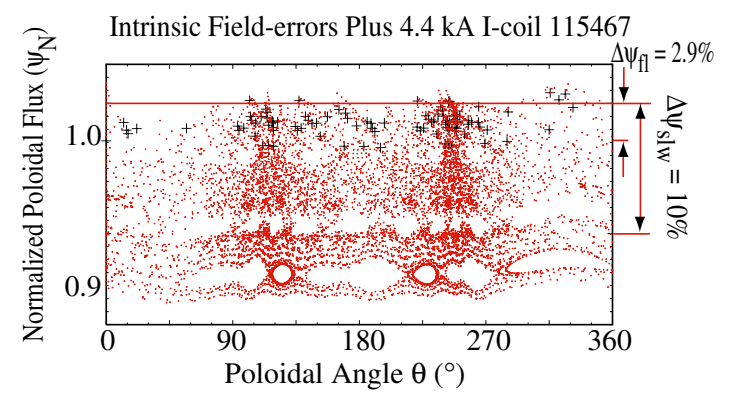

Figure 20. Rectangular Poincaré plot showing how the I-coil perturbation in discharge 115467 changes the pedestal structure owing to the field-errors shown in figure 19.

as well. Another significant figure of merit for these stochastic layers is the mixing width. Here, field lines move randomly between higher and lower values of $\psi_{\mathrm{N}}$ (i.e. the KAM surfaces are broken between resonant island layers) but the excursions in $\psi_{\mathrm{N}}$ are not large enough to take these field lines (excluding those in the flux loss region) across the unperturbed separatrix. The interaction of these two regions can be quite complex and is the subject of a newly emerging research activity on resonant and non-resonant homoclinic tangles in tokamaks $[17,18]$. The collisionality of the plasma in these two regions is of particular importance for understanding transport and stability physics. In the flux loss region high collisionality moves heat and particles perpendicular to the field lines and competes with parallel transports along the 'open' field lines. In the mixing layer, collisions drive particles and energy from one field line to another allowing leakage between field lines sampling larger $\psi_{\mathrm{N}}$ domains with those sampling smaller $\psi_{\mathrm{N}}$ domains when these field lines come into close proximity.

The other significant feature seen in figure 19 is the resonant island chains that fill a large fraction of the pedestal volume. For the case shown we see a relatively large island chain on the $q=3$ surface. Referring to figure 18 and scaling the $m, n=3,1$ and 9, 3 field-error components down to this surface, we see that this island chain results from resonant perturbation of order $1 \mathrm{G}$ and that there is a clear $m=9$ modulation superimposed on the basic $m=3$ island structure. The higher $k_{\theta}$ modulations indicate that the $m=9$ perturbation field is slightly smaller in magnitude than the $m=3$ component of the perturbation on this surface, as indicated by the mode spectra in figure 18 . We also note several small island chains between $\psi_{\mathrm{N}}=0.90$ and 0.95 . These are due to the $m, n=10,3$ and 11,3 resonances shown in figure 18. A larger $m, n=4,1$ island chain is also seen just inside the boundary of the flux loss region. Using figure 18 we can also see that when the I-coil perturbation is turned on in these discharges it will dominate the structure of the $q=3$ and $q=10 / 3$ island chains. It will also contribute about equally to the $q=11 / 3$ island chain produced by the $n=3$ field-error and will have a relatively minor effect on the $q=4$ chain which is dominated by the $m, n=4,1$ fielderror component. These effects are clearly shown in figure 20, where the I-coil field has been added to the field-error results shown in figure 19. There is also a relatively small increase in $\Delta \psi_{\mathrm{fl}}$ and $\Delta \psi_{\mathrm{slw}}$ with the I-coil perturbation (here, the field line lost to the target plate, ' + ', makes 98 toroidal transits). It is interesting to note that the properties of the stochastic flux loss and mixing region are rather modestly affected by the I-coil perturbation field, whereas the resonant island chains, particularly those near the top of the pedestal are noticeably altered by the addition of the I-coil field. In particular, the higher $k_{\theta}$ structures are significantly enhanced.

Since the experimental data obtained during the $q_{95}$ scans demonstrated a relatively sharp effect on the ELM suppression, understanding how the high $k_{\theta}$ structures change with respect to the stochastic properties of the region is of considerable interest. Thus, a series of field line simulations were done in this configuration. A sampling of the result from these simulations is shown in figure 21 . Here, we see that the most obvious change in the pedestal structure is the location and size of the resonant islands. We also note that $\Delta \psi_{\mathrm{fl}}$ is relatively small with $q_{95}=3.78$ and 3.95 compared with 3.36 and 4.46 . Finally, we see that the alignment and density of high $k_{\theta}$ island structures with the inner part of the pedestal are maximized in the $q_{95}=3.78$ and 3.95 cases. We refer to this effect as a local peak in the density of high $k_{\theta}$ states across the pedestal. Using the entire data set from these simulations we evaluate the relative weight of the density of high $k_{\theta}$ states compared with the variation in $\Delta \psi_{\mathrm{ff}}$ with $q_{95}$. These results are summarized in figure 22, where we see quite clearly that the density of states between $\psi_{\mathrm{N}}=0.86$ and 0.97 (the peak pressure gradient region of the pedestal profile) is a maximum at $q_{95} \sim 3.7$ and $\Delta \psi_{\mathrm{fl}}$ has a relative minimum at this point.

We also note that, unlike previous stochastic boundary experiments in low power limiter confinement regimes [10], when magnetic perturbations of the type discussed in this paper are applied to diverted H-mode plasmas, there is a substantial change in the pedestal plasma dynamic to the newly imposed topology that is not seen in highly collisional L-mode plasmas with otherwise identical shape parameters. This fact explicitly highlights the importance of understanding the plasma response to the external magnetic perturbations when interpreting experiments of the type discussed in this paper.

\section{Discussion and implications for future devices}

In addition to developing a better understanding of the basic physics controlling the dynamics of the pedestal region in high power tokamaks, the DIII-D ELM control experiments are motivated by an appreciation of the fact that the next generation of tokamaks will have to rely on such systems to achieve sustained fusion gains of $\sim 10$. The results discussed here provide considerable optimism for developing a system based on edge RMP for ELM control in ITER. Nevertheless, we do not yet have a fundamental theory for the physics underlying the suppression mechanism and thus need to exercise caution in projecting the effectiveness of such an approach to future tokamaks. Our goal here is to use data from DIII-D to develop suppression models that will assist in scaling this approach to burning plasma tokamaks. As such, in this section we briefly discuss the implications suggested by the kind of magnetic pedestal topology found with the field line integration modelling in the previous section and indicate how such a topology may affect the pedestal dynamics in a way that is consistent with the experimental observations.

First we note that this paper focuses primarily on $n=3$, odd parity, $0^{\circ}$ phase (with the exception of the low collisionality 


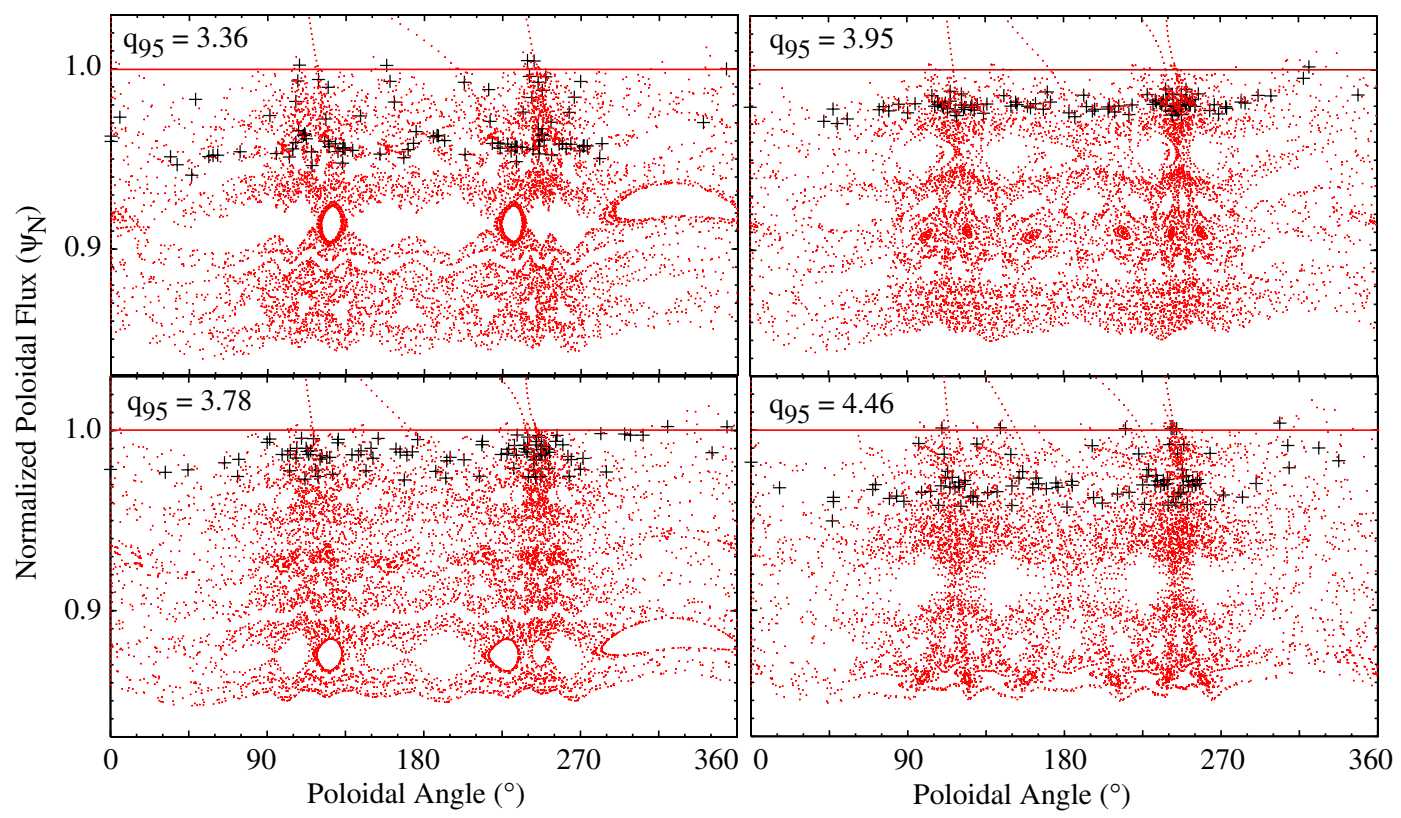

Figure 21. Rectangular Poincaré plots showing changes in the magnetic structure of the pedestal at four values of $q_{95}$ (i.e. $q_{95}=3.36$, 3.78, 3.95 and 4.46) for discharge 115472 with the reference shape as discussed in section 2.3 (figure 15).



Figure 22. The width of the stochastic flux loss layer $\Delta \psi_{\mathrm{fl}}$ at the foot of the pedestal and the locations of the resonant magnetic island chains in $\psi_{\mathrm{N}}$ as a function of $q_{95}$ from field line simulations for the reference discharge shape discussed in section 2.3.

case, where the I-coil was configured for an $n=3$, odd parity, $60^{\circ}$ phase) I-coil experiments, although it should be mentioned that a variety of interesting results also exist from experiments with other I-coil configurations, where there was a clear enhancement of the edge magnetic stochasticity [8,19]. Even though these experiments did not generally produce the same level of ELM suppression as is seen in the $n=3$, odd parity, $0^{\circ}$ phase cases, they did have a significant impact on the pedestal dynamics. Here, it is important to realize that the I-coil current has been limited to $<4.5 \mathrm{kA}$ (the design limit is $7 \mathrm{kA}$ but the power supplies required to reach this limit are not yet available) for all the experiments done to date and that the coil spectrum is only marginally suitable for creating a stochastic flux loss layer of thickness comparable to the pedestal width (the I-coil was not specifically designed for this application). Thus, the properties of the stochastic region are not particularly well suited to testing the original stochastic pressure flattening concept at these lower I-coil current levels; we expect that as the current in the I-coil is increased this situation will be significantly improved.

By changing the parity of the perturbation coil from odd to even, we see a substantial increase in the midplane recycling and interpret this as an indication of a broader stochastic flux loss layer. This observation is consistent with the calculated poloidal mode spectrum of the perturbation coil and field line integration modelling. Additionally, it is found experimentally that by rotating the toroidal phase angle of the odd parity perturbation from $0^{\circ}$ to $60^{\circ}$, there is a substantial increase in the midplane recycling and an unambiguous flattening of the electron pressure and C VI ion pressure profiles [8], each of which is consistent with classical signatures of a broader stochastic flux loss layer. This sensitivity to the toroidal phase angle of the perturbation strongly suggests that non-axisymmetric field-errors in the DIII-D device have a significant effect on the magnetic topology of the pedestal and the ELM dynamics. Although these field-errors have been carefully measured in DIII-D [20], it is possible that they may change with time or that additional components are present that have not been resolved by these measurements. In the moderately stochastic cases (even parity $0^{\circ}$ phase and odd parity $60^{\circ}$ phase), some Type-I ELM suppression is observed but these configurations have yet to be fully explored when the I-coil current can be increased to the $7 \mathrm{kA}$ design limit and the width of the stochastic layer increased to better control the shape of the pedestal profile. A final note, with respect to the effect of the perturbation on the plasma, is that changes in the observations with the toroidal phase angle of the perturbation can, in principle, be related to the rotation of a helical structure with respect to the fixed diagnostic systems. Field line modelling indicates that small helical island structures are present across part of the pedestal, as 
expected in the weakly stochastic regime, and that a helical separatrix structure, referred to as a homoclinic tangle, is formed when the primary separatrix splits into a stable and an unstable manifold owing to magnetic perturbations from both the I-coil and the field-errors [17,18]. Although the ELM suppression data clearly show that the MHD plasma response to the perturbation is both global and uniform in space for the $0^{\circ}$ odd parity case, there are signatures on some diagnostics that quasi-steady-state helical structures are amplified during the I-coil pulse. The nature of these helical structures and their impact on the edge transport and stability of the plasma is an important area of current research, which, as discussed later, may well be related to the suppression mechanism found during the zero degree, odd parity, perturbation experiments.

Field line integration modelling using the TRIP3D code [10] indicates that the best ELM suppression found to date occurs when a weak stochastic magnetic layer is formed across most of the pedestal with a narrow poloidal flux loss region $\left(\sim 3 \%\right.$ in $\left.\psi_{\mathrm{N}}\right)$ at the foot of the pedestal and a relatively large $\left(\sim 3-4 \%\right.$ in $\psi_{\mathrm{N}}$ ) island chain on the $q=3$ surface at the top of the pedestal [8]. The lack of a wide stochastic flux loss region in the TRIP3D modelling is consistent with the relatively small changes observed in the electron pedestal profiles. Further, the lack of any measurable change in any diagnostic signals when the same external perturbation is applied in L-mode suggests that the TRIP3D modelling is incomplete because the plasma response in $\mathrm{H}$-mode is critical for obtaining ELM suppression. There is also evidence that the ELM behaviour depends on the mixing of the applied external perturbation with the intrinsic field-errors in DIII-D, and it is difficult to predict the spectrum and amplitude of such field-errors in future devices. In fact, the sensitivity of the observed ELM behaviour to the toroidal phase of the externally applied $n=3$ perturbation field relative to the intrinsic field-errors, suggests that differences in ELM behaviour between devices might arise from differences in the intrinsic errors in those devices. We also note that even in the best suppression cases, we always observe a few isolated ELMlike events. The characteristics of these events are still under investigation. It seems plausible to assume that if the higher frequency transport induced by the magnetic perturbation is not of sufficient magnitude to maintain a balance between the core energy production rate and power exhaust through the boundary, a net increase in the pedestal pressure will occur and result in isolated impulses equivalent to those of Type-I ELMs.

The results discussed here were obtained over a range of shapes $0.38 \leqslant \delta_{\text {low }} \leqslant 0.73$ and pedestal collisionalities $0.03 \leqslant \nu_{*}^{\text {ped }} \leqslant 0.79$. In addition, the changes observed in the pedestal dynamics when ELMs are suppressed is significantly different with each shape and at each collisionality. This variability should be expected since field line integration studies clearly show that there is a strong dependence in the resonance structure of the pedestal with shape and edge magnetic shear [12]. In particular, the size and shape of the magnetic islands as well as the width of the stochastic flux loss layer are sensitive to the details of the shape, whereas the relative effectiveness of the parallel transport along the open field lines and the rate of flux exchange between stochastic mixing layers in the closed field stochastic region are a strong function of collisionality. These effects also influence the size and position of the resonance window so it is quite reasonable for us to expect to find that the window for the best ELM suppression moves and changes size with different shapes and collisionalities.

Looking only at results from the high triangularity case discussed in section 2.3 (i.e. those summarized by the change in dynamics shown in figure 7) along with the field line integration modelling results discussed for this case in section 3 , we are led to the conclusion that the suppression mechanism is at least partially related to a change in the structure and density of islands across the pedestal when the I-coil is activated. On the other hand, the open field stochastic region can also be expected to play a significant role in the pedestal stability since it has an impact on both the pressure and current profile across the outer $2-3 \%$ of the pedestal. This region has a relatively strong influence on peeling-ballooning stability constraints. Each of these mechanisms is being investigated as a possible explanation for the suppression of large Type-I ELMs in this configuration and will be the focus of future experiments to determine the relative role each may be playing. Thus, as additional current becomes available in the I-coil, experiments will be carried out to assess the relative importance of remnant island versus stochastic layer effects and to test the effectiveness of increasing the coil current on broadening the resonant ELM suppression window with various shapes and collisionalities.

\section{Conclusions}

Fast heat pulses, driven by large Type-I ELMs in the DIII-D divertor, are eliminated for periods approaching nine energy confinement times with small dc currents driven in a simple magnetic perturbation coil (the DIII-D I-coil). All but a few isolated ELM-like events are reproducibly eliminated during a $2 \mathrm{~s}$ pulse. Based on field line modelling, the magnetic perturbation from the coil resonates strongly with the plasma flux surfaces across most of the pedestal region $0.9 \leqslant \psi_{\mathrm{N}} \leqslant$ 1.0 , creating remnant islands surrounded by weakly stochastic field lines. The stored energy, $\beta_{\mathrm{N}}$ and $\mathrm{H}$-mode quality factor are unaffected by the perturbation field. The electron pressure profile, radial electric field and poloidal rotation across the pedestal are also unaltered along with the H-mode transport barrier.

ELMs are replaced by an increase in electron density and magnetic field fluctuations. These fluctuations have a distinct bursty and/or intermittent character and are modulated by a $130 \mathrm{~Hz}$ oscillation with a $2 \mathrm{~ms}$ quiet period followed by a $6 \mathrm{~ms}$ active period. Using the drops in the stored energy with and without the edge RMP and estimates of the time required for the energy drops, implies roughly a factor of 3 reduction in the energy impulse source from the pedestal into the SOL. Particle flux impulses to the divertor target are suppressed by about a factor of 8. In addition, the heat flux to the divertor plate, as inferred from the surface temperature changes of the divertor tile near the strike point, becomes much less impulsive. The peak heat flux on the outer divertor target plates, averaged over many ELM periods, drops by at least a factor of 5 .

Good ELM suppression has been obtained over a wide range of shapes $0.38 \leqslant \delta_{\text {low }} \leqslant 0.73$ and pedestal collisionalities $0.03 \leqslant v_{*}^{\text {ped }} \leqslant 0.79$. Although the basic physics of the suppression mechanism is still under investigation, 
comparisons between the magnetic topology calculated with a field line integration code and experimental observations in one of the configurations studied, suggests that an increase in the density of high $k_{\theta}$ island states may be partially responsible for the suppression of Type-I ELMs but small changes in the stochastic structure at the foot of the pedestal could also be involved. Future experiments, with increased I-coil current, will focus on isolating the relative importance of these two mechanisms.

\section{Acknowledgments}

Work supported by the US Department of Energy under DE-FC02-04ER54698, DE-FG02-04ER54158, DE-AC0494AL85000, W-7405-ENG-48, DE-FG02-89ER53297, DEFG03-01ER54615 and DE-AC05-00OR22725 is gratefully acknowledged.

\section{References}

[1] Loarte A. et al 2003 Plasma Phys. Control. Fusion 451549

[2] Federici G. et al 2003 Plasma Phys. Control. Fusion 451523

[3] Pacher H.D. 1996 ITER Design Description Document ITER No G 17 DDD 1 96-08-21 W2.1, appendix E9, section 1.7
[4] Maddison G.P. et al 2003 Plasma Phys. Control. Fusion 451657

[5] Lang P.T. et al 2003 Nucl. Fusion 431110

[6] Tamai H. et al 1994 J. Nucl. Mater. 220-222 365

[7] Felding S.J. et al 2002 Proc. 28th EPS Conf. on Controlled Fusion and Plasma Physics (Madeira, 2001) vol 25A (European Physical Society) p 1825

[8] Evans T.E. et al 2004 Phys. Rev. Lett. 92 235003-1

[9] Evans T.E. et al $2005 \mathrm{~J}$. Nucl. Mater. 337-339 691

[10] Ghendrih Ph., Grosman A. and Caps H. 1996 Plasma Phys. Control. Fusion 381653

[11] Evans T.E. et al 1987 J. Nucl. Mater. 145-146 812

[12] Evans T.E. et al 2002 Phys. Plasmas 34957

[13] Evans T.E. et al 1992 J. Nucl. Mater. 196-198 421

[14] Jackson G.L. et al 2003 Proc. 30th EPS Conf. on Controlled Fusion and Plasma Physics (St Petersburg, 2003) CD-ROM P-4.47

[15] Evans T.E. and Moyer R.A. 2003 J. Nucl. Mater. 313-316 1282

[16] Gribov Y. 2004 Physics Unit, ITER Naka Joint Work Site, Japan, personal communication

[17] Roeder R.K.W., Rapoport B.I. and Evans T.E. 2003 Phys. Plasmas 103796

[18] Evans T.E. et al 2004 Contrib. Plasma Phys. 44235

[19] Moyer R.A. et al 2005 Edge localized mode control with and edge resonant magnetic perturbation Phys. Plasmas 12 056119

[20] Luxon J.L. et al 2003 Nucl. Fusion 431813 